\title{
Laudio for Prof. Dr. Ryoji Noyori
}

\author{
Dedicated Issue of SYNLETT
}

This issue of Synlett celebrates Professor Ryoji Noyori for his legendary contributions to synthetic organic chemistry. Professor Noyori is considered the premier synthetic chemist of our times and has made splendid contributions to the creation of the organic chemistry of the $21^{\text {st }}$ century. During his career, his research has ranged broadly and has included new synthetic methods, the synthesis of biologically active compounds, stereoselective reactions, new reaction media, organometallic chemistry and homogeneous catalysis. Innovation and creativity are the hallmarks of Professor Noyori's scientific achievements in all of these areas. Applications of his original and versatile chemistry have allowed him and other scientists to realize truly efficient syntheses of organic molecules of theoretical and practical importance.

Of particular note is his brilliant work in the development of asymmetric catalysis. In 1966, he reported the first example of a homogeneous asymmetric catalysis by well-defined transition metal complexes when he discovered the enantioselective cyclopropanation of olefins with diazoacetates in the presence of a chiral Schiff base-Cu(II) complex. In the early 1980's, Professor Noyori devised a number of transition metal catalysts containing a new atropisomeric chiral diphosphine, BINAP, which have proven remarkably effective in a series of asymmetric transformations. This invention provided a major breakthrough in stereoselective organic synthesis. In particular, his discovery of BINAP-Ru(II) complex catalysts led to stereoselective hydrogenation of wide-ranging olefinic and ketonic substrates. A variety of terpenes, vitamins, $\beta$-lactam antibiotics, $\alpha$ - and $\beta$-amino acids, isoquinoline alkaloids, and other compounds of biological interest such as carnitine and antiinflammatory naproxen are accessible using this hydrogenation method. In 1995, Professor Noyori found a new chiral diphosphine/diamine Ru complex catalyst that effects a long-sought carbonyl-selective hydrogenation without affecting a coexisting olefinic or acetylenic linkage. This new method allows enantioselective hydrogenation of a series of simple olefinic and aromatic ketones to the secondary alcohols with high ee's. This new hydrogenation is very clean, operationally simple, economical, environmentally friendly, and can be conducted on any scale with a substantially high substrate/catalyst ratio and very high substrate concentration in organic solvents. The BINAP-Ru catalyzed hydrogenation of acetol to $(R)$-1,2-propanediol is now used for the large-scale synthesis of levofloxacin, an antibacterial quinolone. The reaction of racemic methyl $\alpha$-(benzamidomethyl)acetoacetate (dynamic kinetic resolution) is also being utilized in the industrial production of a chiral $\beta$-hydroxy ester, which serves as a common intermediate for the synthesis of carbapenem antibiotics. He also invented phosphine- and nonphosphine-based chiral $\mathrm{Ru}(\mathrm{II})$ catalysts for efficient transfer hydrogenation of ketones with 2-propanol or a formic acid triethylamine mixture as hydrogen donors.

Professor Noyori's BINAP-Rh(I) complexes have proved to effect highly enantioselective isomerization of diethylgeranylamine to $(R)$-citronellal enamine. This asymmetric reaction working on a 9-ton scale is a key step in the industrial synthesis of (-)-menthol, the world's largest application of asymmetric catalysis.

In 1986, Professor Noyori achieved the first highly enantioselective addition of dialkylzincs to aldehydes using a catalytic quantity of a chiral $\beta$-dialkylamino alcohol, where alkylation products with high enantiomeric excesses are accessible with the partially resolved chiral ancillary. The mechanism of this striking chirality amplification was elucidated at the molecular structure level in 1989. This is based on the self- and nonself recognition of asymmetric catalysts, and the phenomenon has been proven to be very general.

Chiral binaphthol-modified lithium aluminum hydride reagents, elaborated by Professor Noyori in the late 1970's, exhibit extremely high enantioselection in the reduction of an extensive array of prochiral carbonyl compounds. This asymmetric reduction greatly facilitated the current commercial synthesis of prostaglandins. His selective organometallic methodologies using organocopper, -tin, and -zinc reagents, combined with his asymmetric reduction procedure, realized the long-sought three-component coupling synthesis of prostaglandins, which allows flexible preparation of both natural and unnatural products. Some notable applications include the controlled synthesis of therapeutically significant isocarbacyclin, a stable prostacyclin analogue, and the development of APNIC, a photoaffinity probe for a prostacyclin receptor.

Professor Noyori's contribution to organic chemistry is not limited to asymmetric synthesis. In 1994, he demonstrated for the first time the remarkable utility of supercritical carbon dioxide as a medium for homogeneous catalysis by accomplishing its own fixation by $\mathrm{Ru}$ catalyzed hydrogenation to produce formic acid, methyl formate, and dimethylformamide in extremely high turnover numbers, up to $420,000 \mathrm{~mol} / \mathrm{mol} \mathrm{Ru}$.

Professor Noyori's intense interest in organometallic chemistry drove him to devise novel methods in nucleic acid synthesis as well. Thus, his invention in solution-phase chemistry includes $\mathrm{O}$-selective phosphorylation of nucleosides without $\mathrm{NH}_{2}$ blocking, efficient formation of internucleotide linkage, and nonaqueous oxidation of phosphites, which results in a straightforward entry to biologically significant 2',5'-linked oligoadenylates, branch-type oligoribonucleotides, and other artificial analogues. In 1990, he achieved the first, very efficient synthesis of solidanchored DNA oligomers via organopalladium chemistry utilizing allylic protective groups.

In his early days in Nagoya, Professor Noyori found the iron carbonyl/polybromo ketone reactions which allow direct construction of five- and seven-membered carbocycles in a [3+2] and $[3+4]$ manner, respectively. The utility has been amply demonstrated by the synthesis of troponoids, C-nucleosides, and other natural carbocycles. He was intrigued by the chemistry of sframed strained hydrocarbon systems in the presence of $\mathrm{Ni}(0)$ complexes and proposed the significance of metallacycle mechanisms. He also elucidated the behavior of epoxides and biologically important endoperoxides under the influence of transition metal complexes.

Organosilicon chemistry is another of his interests. Professor Noyori demonstrated that trimethylsilyl triflate acts as a versatile catalyst in numerous organic reactions including aldol-type reaction and glycosylation reactions. He developed the chemistry of tris(dialkylamino)sulfonium enolates (ion-paired enolates rather than ordinary Lewis acid-interacting enolates) undergoing a uniquely stereoselective aldol reaction.

On behalf of the extended family of Noyori disciples and his many friends and colleagues worldwide, I wish him continued success, satisfaction and happiness and acknowledge with heartfelt gratitude his enduring example of creativity, scholarship and humanity.

Hisashi Yamamoto

Editor of special issue 


\section{Special Issue}

\section{Dedicated to Ryoji Noyori}

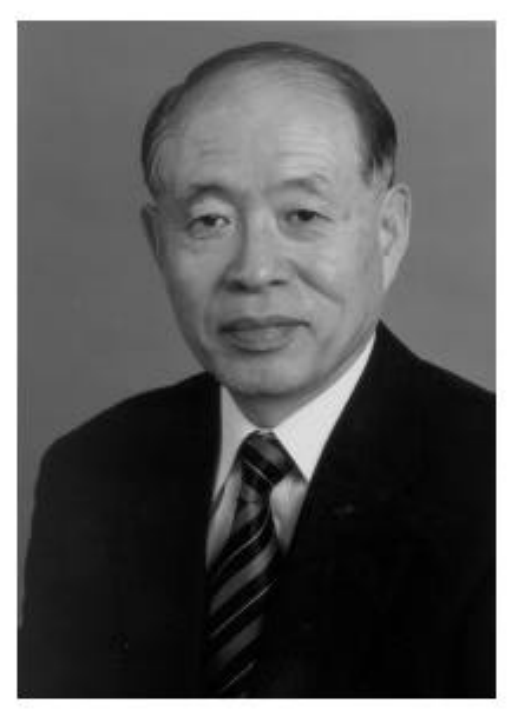

Born in Hyogo Prefecture, Japan; September 3, 1938.

Bachelor, Kyoto University, 1961. Master, Kyoto University, 1963. Ph.D., Kyoto University, 1967. Postdoctoral Fellow, Harvard University, 1969-1970.

Kyoto University: Instructor, 1963-1968. Nagoya University: Associate Professor, 1968-1972. Professor, 1972-present. Director, Chemical Instrument Center, 1979-1991. Dean, Graduate School of Science, 1997-1999. Director, Research Center for Materials Science, 2000-present.

The Research Development Corporation of Japan: Director of the ERATO Molecular Catalysis Project, 1991-1996. Ministry of Education, Science, Sports and Culture: Science Advisor, 19921996. Member of Scientific Council, 1996-2001. Ministry of Education, Culture, Sports, Science and Technology: Member of Scientific Council, 2001-present. Kyushu University: Professor (adjunct) 1993-1996. Japan Society for the Promotion of Science: Committee Chairman, Research for the Future Program on "Advanced Processes", 1996-present. The Society of Synthetic Organic Chemistry, Japan: Vice President, 1994- 1996. President, 1997-1999. The Chemical Society of Japan: President-Elect, 2001.

Honorary Degrees: Technische Universität München (1995). Universite de Rennes 1 (2000).

Fellowship and Membership: Fellow, American Association for the Advancement of Science (1996). Honorary Member, Chemical Society of Japan (1998). Honorary Fellow, Royal Society of Chemistry (London) (2000).

Honored as a Person of Cultural Merit (1998) and awarded the Order of Culture (2000) from the Japanese Emperor/Government. Awards: The Chemical Society of Japan Award for Young Chemists (1972). The Matsunaga Prize (Matsunaga Memorial Foundation, Japan, 1978). The Chunichi Cultural Prize (Chunichi Newspaper Co., Japan, 1982). The Chemical Society of Japan Award (1985). The Naito Foundation Research Prize (Naito Science Foundation, Japan, 1988). The Fluka Prize, Reagent of the Year (Fluka Chemie AG, Switzerland, 1989). The Centenary Medal (The Royal Chemical Society, London, 1990). The Toray Science \& Technology Prize (Toray Science Foundation, Japan, 1990). The Merck-Schuchardt Chair (BOSS Symposium, Belgium, 1990). The J. G. Kirkwood Award (Yale University, 1991). The Asahi Prize (Asahi Culture Foundation, Japan, 1992). Tetrahedron Prize for Creativity in Organic Chemistry (Pergamon Press, UK, 1993). The Keimei Life Science Prize (Keimei Foundation, Japan, 1994). The Japan Academy Prize (1995). The Arthur C. Cope Scholar Award (ACS, 1996). Bonn Chemistry Award (University of Bonn and Pinguin Foundation, 1996). The Arthur C. Cope Award (ACS, 1997). The Chirality Medal (International Symposium on Chiral Discrimination, 1997). The George Kenner Award (University of Liverpool, 1997). The King Faisal International Prize for Science (Saudi Arabia, 1999). The Cliff S. Hamilton Award (University of Nebraska, 1999). ISI Citation Laureate Award (ISI/Thomson Scientific Inc., 2000). The Special Award (The Society of Synthetic Organic Chemistry, Japan, 2001). The Wolf Prize in Chemistry (Wolf Foundation, Israel, 2001). The Roger Adams Award in Organic Chemistry (ACS, 2001). 
V

To the readers and contributors of synlett,

I am grateful to all of my colleagues worldwide who have contriderted their excellent scientific achievemeats to this special iss me of syolett.

Organic cherristing is beautiful, exciting, and beneficial to humankind. I am very proud that I have devoted myself for over four decades to synthetic organic chemist, an extremely important scientific realm. Substances and material determine the quality of life. State-of-the-aut chemical synthesis allows for the construction of architecturally and functionally highly complex molecule. In view of the inherent significance of om science, it is now important to make its methods more practical. If the efficiency of synthesis and catalysis would increase by two orders of magnitude, fro instance, enormous opportune ties would open up in chemistry and related industry. Furthermore, chemical syn thess provides a logical bans for progress in the biosciences and material sciences, where molecules engineering is cuncially important.

Om activities should increasingly become creative and dynamic. I believe that furthering om understanding of precise biological mechanism e by chemists will leads to the discovery of rational, more effective pharmaceuticals in the port-genome en. A range of molecule -bared materials will be invented by synthetic chemists only if they have deep insight into applied physics. This cues is fuel of opportunities for synthetic chemistry. In addition, om endeavors should further be diced toward solving a range of existing, or even unforeseen, social and global issues associated with health, food, material, energy, and environments, among others.

I to hope that om younger generation will create new values in the let century though chemistry.

Raven

Felomany 9, 2001

Nagoya 